\title{
Dynamics of resource production and utilisation in two-component biosphere-human and terrestrial carbon systems
}

\author{
M. R. Raupach \\ CSIRO Marine and Atmospheric Research, Canberra, ACT 2601, Australia \\ Received: 16 June 2006 - Published in Hydrol. Earth Syst. Sci. Discuss.: 17 August 2006 \\ Revised: 29 November 2006 - Accepted: 9 Februsray 2007 - Published: 21 February 2007
}

\begin{abstract}
This paper analyses simple models for "production-utilisation" systems, reduced to two state variables for producers and utilisers, respectively. Two modes are distinguished: in "harvester" systems the resource utilisation involves active seeking on the part of the utilisers, while in "processor" systems, utilisers function as passive material processors. An idealised model of biosphere-human interactions provides an example of a harvester system, and a model of plant and soil carbon dynamics exemplifies a processor system. The biosphere-human interaction model exhibits a number of features in accord with experience, including a tendency towards oscillatory behaviour which in some circumstances results in limit cycles. The plant-soil carbon model is used to study the effect of random forcing of production (for example by weather and climate fluctuations), showing that with appropriate parameter choices the model can flip between active-biosphere and dormantbiosphere equilibria under the influence of random forcing. This externally-driven transition between locally stable states is fundamentally different from Lorenzian chaos. A behavioural difference between two-component processor and harvester systems is that harvester systems have a capacity for oscillatory behaviour while processor systems do not.
\end{abstract}

\section{Introduction}

We are by now accustomed to the idea of Planet Earth as a single entity including interacting geophysical, biotic and human constituent systems. Among the attributes of the earth system and its components is a propensity for autonomous dynamism. Parts of the earth system follow temporal trajectories which can exhibit a wide range of behaviours growth, decay, quasi-periodic cycling, relatively sudden flips

Correspondence to: M. R. Raupach

(michael.raupach@csiro.au) between alternative states, and apparently random fluctuations. These dynamic behaviours are sometimes easily attributable to external drivers, but often they are not. Examples include climate phenomena from interannual variability to ice ages; the dynamics of ecosystems, including population cycles, explosions and crashes; and the dynamics of social-ecological systems involving humans, such as boombust cycles and societal collapses associated with resource exploitation and depletion.

This paper focuses on the dynamical properties of parts of the earth system which are governed by the linked production and utilisation of resources. The broad aim is to identify basic system attributes which underlie commonly observed dynamical behaviours such as cycles and threshold transitions. For this purpose, production-utilisation systems will be idealised to just two components or state variables, respectively describing the producers and the utilisers. Within this framework, two (not always disjoint) modes for the production-utilisation interaction will be contrasted. In the first mode, resource utilisation occurs by active, often goal-seeking behaviour on the part of the utilisers; such systems can be characterised as "harvester" systems. Examples include prey-predator systems and (at a high level of abstraction) the biosphere-human system. In the second mode, the utilisers process resources which they receive largely passively, to achieve closed material cycles (through loops including the world outside the system under study) or to prevent accumulation of waste in the production side of the system. Examples include water, carbon and nutrient cycling in terrestrial systems, and the production and disposal of goods in human societies. Such systems can be characterised as "processor" systems. It will be shown by example how these two modes for production and utilisation lead to different characteristic dynamical properties.

For this comparative exercise, two systems are studied with highly simplified models consisting of just two equations - one for producers, one for utilisers. An idealised

Published by Copernicus GmbH on behalf of the European Geosciences Union. 
model of biosphere-human interactions is used as an example of a harvester system, and a similarly idealised model of plant and soil carbon dynamics provides a model of a processor system. The formal approach is based on the theory of dynamical systems, drawing from a well-established body of applied mathematics (e.g. Drazin, 1992; Glendinning, 1994) and particularly from applications in mathematical ecology (e.g. Gurney and Nisbet, 1998; Kot, 2001).

The plan of the paper is as follows. In Sect. 2, some necessary aspects of dynamical systems theory are summarised briefly. Sections 3 and 4 apply this general framework to a two-equation model of biosphere-human interactions, showing how even this minimal model can reproduce features of biosphere-human systems which are recognisable from qualitative experience. In Sect. 5, a comparable analysis is made of a two-equation model for interactions between plant and soil carbon. Section 6 draws conclusions.

\section{Dynamical systems theory}

Consider a producer-utiliser system with two state variables $\left(x_{1}, x_{2}\right)$, governed by

$$
\begin{aligned}
& d x_{1} / d t=f_{1}(\mathbf{x})=g_{1}(\mathbf{x})-g_{2}(\mathbf{x})-k_{1} x_{1} \\
& d x_{2} / d t=f_{2}(\mathbf{x})=r g_{2}(\mathbf{x})-k_{2} x_{2}
\end{aligned}
$$

where $x_{1}(t)$ is the density of resource producers, $x_{2}(t)$ the density of utilisers, $g_{1}(\mathbf{x})$ is the primary production flux into the $x_{1}$ pool, $g_{2}(\mathbf{x})$ is the resource utilisation flux from the $x_{1}$ pool into the $x_{2}$ pool, $r$ is the efficiency for conversion of $x_{1}$ into $x_{2}, k_{i}(i=1,2)$ is a first-order decay rate, and $f_{i}(\mathbf{x})=$ $d x_{i} / d t$ is the net input flux to the $x_{i}$ pool. The equations are coupled by the dependence of the fluxes $g_{1}(\mathbf{x})$ and $g_{2}(\mathbf{x})$ on both state variables $\left(x_{1}, x_{2}\right)$. The equation system can be written in matrix form as

$$
\begin{aligned}
& d \mathbf{x} / d t=\mathbf{f}(\mathbf{x})=\mathbf{R} \cdot \mathbf{g}(\mathbf{x})-\mathbf{K} \cdot \mathbf{x} \\
& \mathbf{x}(t)=\left(\begin{array}{l}
x_{1}(t) \\
x_{2}(t)
\end{array}\right), \quad \mathbf{f}(\mathbf{x})=\left(\begin{array}{c}
f_{1}(\mathbf{x}) \\
f_{2}(\mathbf{x})
\end{array}\right), \\
& \mathbf{g}(\mathbf{x})=\left(\begin{array}{c}
g_{1}(\mathbf{x}) \\
g_{2}(\mathbf{x})
\end{array}\right), \quad \mathbf{R}=\left(\begin{array}{cc}
1 & -1 \\
0 & r
\end{array}\right), \quad \mathbf{K}=\left(\begin{array}{cc}
k_{1} & 0 \\
0 & k_{2}
\end{array}\right)
\end{aligned}
$$

Models are needed for the production and utilisation fluxes, $g_{1}(\mathbf{x})$ and $g_{2}(\mathbf{x})$. For the production flux, some common possibilities are:

$$
\begin{array}{ll}
\text { model P0: } & g_{1}(\mathbf{x})=p_{1} \\
\text { model P1: } & g_{1}(\mathbf{x})=p_{1} x_{1} \\
\text { model P2: } & g_{1}(\mathbf{x})=p_{1}\left(\frac{x_{1}}{x_{1}+q_{11}}\right) \\
\text { model P3: } & g_{1}(\mathbf{x})=p_{1}\left(\frac{x_{1}}{x_{1}+q_{11}}\right)\left(\frac{x_{2}}{x_{2}+q_{12}}\right)
\end{array}
$$

In model P0 the production flux $g_{1}$ is constant, while in model $\mathrm{P} 1$ it is proportional to the producer biomass $x_{1}$. In model P2, $g_{1}$ has a saturating dependence on $x_{1}$ of MichaelisMenten or Holling Type II form (Gurney and Nisbet, 1998) with scale $q_{11}$, so that production depends linearly on $x_{1}$ when $x_{1}<<q_{11}$ and is independent of $x_{1}$ when $x_{1}>>q_{11}$. In model P3, $g_{1}$ has a saturating dependence on $x_{1}$ as for model $\mathrm{P} 2$, together with a similar dependence on the utiliser as a symbiont, $x_{2}$, with scale $q_{12}$.

For the utilisation flux $g_{2}(\mathbf{x})$, common possibilities are

model U0: $\quad g_{2}(\mathbf{x})=p_{2} x_{1}$

model U1: $g_{2}(\mathbf{x})=p_{2} x_{2} x_{1}$

model U2: $g_{2}(\mathbf{x})=p_{2} x_{2}\left(\frac{x_{1}}{x_{1}+q_{21}}\right)$

In model U0, $g_{2}$ is independent of utiliser level $\left(x_{2}\right)$ and depends only on resource availability $\left(x_{1}\right)$. Models U1 and U2 both assume a dependence of $g_{2}$ on $x_{2}$. The notation in Eqs. (3) and (4) is that $p_{i}$ is a scale for the overall magnitude of the flux $g_{i}$, and $q_{i j}$ is a scale for the modification of $g_{i}$ by state variable $x_{j}$ (so $q_{i j}$ appears in the equation for $g_{i}$ and has the dimension of $x_{j}$ ).

The distinction between two-component harvester and processor systems, as characterised above, can be made formal through the model for $g_{2}(\mathbf{x})$. In processor systems, where the utilisers receive recources passively, $g_{2}(\mathbf{x})$ is independent of $x_{2}$ and depends only on $x_{1}$ (as in model U0); in harvester systems $g_{2}(\mathbf{x})$ depends on both $x_{1}$ and $x_{2}$ (as in models $\mathrm{U} 1$ and $\mathrm{U} 2$ ).

A particular model is specified by the parameterisations for the production and utilisation fluxes from the above possibilities (or others). For instance, the wellknown Lotka-Volterra equations $\left(d x_{1} / d t=p_{1} x_{1}-p_{2} x_{2} x_{1}\right.$, $\left.d x_{2} / d t=p_{2} x_{2} x_{1}-k_{2} x_{2}\right)$ for predator-prey dynamics (Lotka 1920; Volterra, 1926), are of the class P1U1. Several cases, including P0U1 and P2U1, are analysed by Gurney and Nisbet (1998) and Kot (2001).

The solution of the system is a trajectory $\mathbf{x}(t)$ in state $(\mathbf{x})$ space, from a given initial state $\mathbf{x}(0)$ at time $t=0$, with given models for $g_{1}$ and $g_{2}$ and with given parameters $\left(r, k_{i}, p_{i}\right.$, $\left.q_{i j}, \ldots\right)$. Much of the behaviour of this solution is determined by the equilibrium points $\left(\mathbf{x}^{Q}\right.$, denoted by a superscript $Q$ ) at which $d \mathbf{x} / d t=\mathbf{f}(\mathbf{x})=0$, and by the local stability of the trajectories around these points (Drazin, 1992; Glendinning, 1994; Casti, 1996, 2000). The existence of equilibrium points is governed by the nonlinear equation

$\mathbf{f}\left(\mathbf{x}^{Q}\right)=\mathbf{R} \cdot \mathbf{g}\left(\mathbf{x}^{Q}\right)-\mathbf{K} \cdot \mathbf{x}^{Q}=0$

which is satisfied when $\mathbf{x}^{Q}$ is an equilibrium point. The stability of $\mathbf{x}^{Q}$ is determined by the linearised system

$d \mathbf{x}^{\prime} / d t=\mathbf{J} \cdot \mathbf{x}^{\prime}$

where $\mathbf{J}=J_{i j}$ is the Jacobian matrix $\left(\partial f_{i} / \partial x_{j}\right)$ of the vector function $\mathbf{f}(\mathbf{x})$, and $\mathbf{x}^{\prime}=\mathbf{x}-\mathbf{x}{ }^{Q}$ is a perturbation about $\mathbf{x}^{Q}$. The eigenvalues $\left(\lambda_{i}\right)$ of $\mathbf{J}$ are solutions of the characteristic equation $\operatorname{Det}(\mathbf{J}-\lambda \mathbf{I})=0$. If all eigenvalues of $\mathbf{J}\left(\right.$ evaluated at $\left.\mathbf{x}^{Q}\right)$ 
have negative real parts, then $\mathbf{x}^{Q}$ is stable (so that trajectories near $\mathbf{x}^{Q}$ converge to $\mathbf{x}^{Q}$ as $t \rightarrow \infty$ ), and if at least one $\lambda_{i}$ has a positive real part, then $\mathbf{x}^{Q}$ is unstable (so that an infinitesimal disturbance from $\mathbf{x}^{Q}$ causes trajectories to diverge from $\mathbf{x}^{Q}$ as $\left.t \rightarrow \infty\right)$. The imaginary parts of $\lambda_{i}$ determine whether the solutions near $\mathbf{x}^{Q}$ have oscillatory components.

For the two-dimensional system of Eq. (1), the Jacobian is (with $\partial_{j} g_{i}=\partial g_{i} / \partial x_{j}$ ):

$\mathbf{J}=\left(\begin{array}{cc}\partial_{1} g_{1}-\partial_{1} g_{2}-k_{1} & \partial_{2} g_{1}-\partial_{2} g_{2} \\ r \partial_{1} g_{2} & r \partial_{2} g_{2}-k_{2}\end{array}\right)$

For this (or any) two-dimensional system, the characteristic equation for the eigenvalues of $\mathbf{J}$ is

$\lambda^{2}-(\operatorname{Tr} \mathbf{J}) \lambda+(\operatorname{Det} \mathbf{J})=0$

It is well known (Drazin 1992 p. 170-176) that for twodimensional systems the main options for the stability of an equilibrium point $\mathbf{x}^{Q}$ are as follows: if both roots $\left(\lambda_{1,2}\right)$ of Eq. (8) are real and negative (positive), then $\mathbf{x}^{Q}$ is a stable (unstable) node: nearby trajectories converge to (diverge from) $\mathbf{x}^{Q}$ along non-spiralling curves. If both roots $\lambda_{1,2}$ are complex with negative (positive) real parts, then $\mathbf{x}^{Q}$ is a stable (unstable) focus or spiral point: nearby trajectories spiral inward to (outward from) $\mathbf{x}^{Q}$. If the roots $\lambda_{1,2}$ have real parts of opposite sign, then $\mathbf{x}^{Q}$ is a saddle point: nearby trajectories are hyperbolic. A saddle point is unstable in general, except for approach along particular directions. These conditions are equivalent to the following:

$$
\begin{array}{ll}
\mathbf{x}^{Q} \text { is stable if } & (\text { Det } \mathbf{J})>0 \text { and }(\operatorname{Tr} \mathbf{J})<0 \\
\mathbf{x}^{Q} \text { is unstable if } & (\operatorname{Det} \mathbf{J})>0 \text { and }(\operatorname{Tr} \mathbf{J})>0 \\
\mathbf{x}^{Q} \text { is a saddle if } & (\text { Det } \mathbf{J})<0
\end{array}
$$

The spiral (oscillatory) tendency of the local trajectories around $\mathbf{x}^{Q}$ is determined by the discriminant $(D)$ of the left side of Eq. (8):

$D>0 \quad$ (stable node: nonspiral)

$D<0$ (stable focus: spiral)

with $\quad D=(\operatorname{Tr} \mathbf{J})^{2}-4 \operatorname{Det} \mathbf{J}$

For two-component processor systems as defined above, $\partial_{2} g_{2}=0$ and $D=\left(\partial_{1} g_{1}-\partial_{1} g_{2}-k_{1}+k_{2}\right)^{2}+4\left(\partial_{1} g_{2}\right)\left(\partial_{2} g_{1}\right) r$. Provided $\partial_{1} g_{2} \geq 0$ and $\partial_{2} g_{1} \geq 0$, as in all examples above, $D$ is positive. In these conditions, oscillatory (spiralling) behaviour is not possible.

\section{Biosphere-human interactions: basic model}

As an example of a producer-utiliser system of the harvester type, we consider a minimal model of biospherehuman interactions in which the biosphere acts as producer and humans as utilisers. The interaction between humans and the natural biosphere that sustains them clearly involves a vast range of biophysical, economic, social and cultural processes which together have shaped human populations diverse ways determined by both biogeographical circumstances and contingent history (Flannery, 1994; Diamond, 1991, 1997, 2005). It goes without further emphasis that a two-equation model cannot capture even a fraction of this richness. Nevertheless, even such a simple model is capable of discerning some broad patterns.

The state variables are the biomass $b(t)$ and human population $h(t)$ in a specified region. We first consider a very simple formulation in which $b(t)$ and $h(t)$ are governed by

$\begin{aligned} d b / d t & =p-c b h-k b \\ d h / d t & =r(c b h-m h)\end{aligned}$

where $p$ is a constant primary biomass production flux, $c$ the rate of extraction of biomass per human; $k$ the rate of decay of biomass by respiration, $m$ the maintenance biomass requirement per unit time per human, and $r$ the fractional growth rate of human population per unit biomass surplus. The model assumes that the growth rate of $h$ depends on the difference $(c b h-m h)$ between the extraction of biomass by harvest $(c b h)$ and the biomass per unit time required to maintain the human population $(m h)$. This difference is a surplus production measured in biomass units, leading to population increase (decrease) at rate $r$ when the surplus $(c b h-m h)$ is positive (negative). This model is a special case of Eqs. (1) to (4) with production and harvest models of the class POU1 and variable substitutions $\left(x_{1}, x_{2}\right) \rightarrow(b, h),\left(g_{1}, g_{2}\right) \rightarrow(p, c b h)$, and $\left(k_{1}, k_{2}\right) \rightarrow(k, r m)$. Assumptions in this highly simplified model are that there is no transfer of either $b$ or $h$ across the boundaries of the model region, and also that biomass production $(p)$ does not depend directly on $h$, for instance by technological innovation (see Wirtz and Lemmen (2003) for a model in which there is a dependence of $p$ on $h$ ).

Given its idealisations and restrictions, the model can be interpreted in two ways. First, $b$ and $h$ can be regarded strictly as biomass and human population, respectively. This view is relevant to interactions between isolated, homogeneous human populations and their environments. A second, broader view regards $b$ as "renewable natural capital" and $h$ as "human capital". In this case the model may have some applicability to technologically advanced societies where increase in human capital continues unchecked even though human populations are stabilising or declining. In the context of farm management, a model with some similarities to the present $(b, h)$ model has been proposed by Fletcher et al. (2006), with $b$ and $h$ interpreted in this way.

The model has three independent dimensional metrics, biomass $[\mathrm{B}]$, humans $[\mathrm{H}]$ and time $[\mathrm{T}]$. There are five parameters $(p, c, k, m$ and $r)$, with units $p\left[\mathrm{BT}^{-1}\right], c\left[\mathrm{H}^{-1} \mathrm{~T}^{-1}\right]$, $k\left[\mathrm{~T}^{-1}\right], m\left[\mathrm{BH}^{-1} \mathrm{~T}^{-1}\right]$ and $r\left[\mathrm{HB}^{-1}\right]$. Dimensional analysis (Bridgman 1931; Huntley 1967) then shows that the system has two $(=5-3)$ independent dimensionless groups. These 

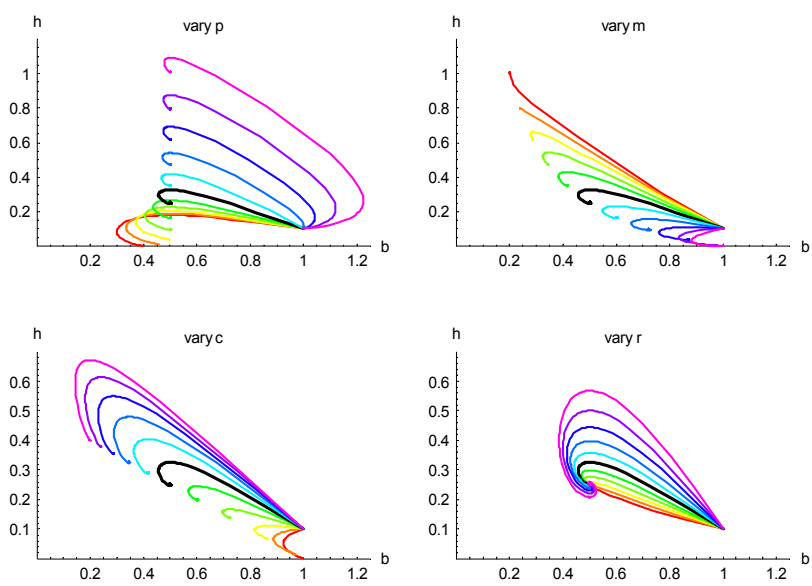

Fig. 1. Trajectories $(b(t), h(t))$ of the basic biosphere-human model on the $b h$ plane, with different curves showing variation of (top left) primary production $p$; (top right) human maintenance requirement $m$; (bottom left) extraction rate $c$; (bottom right) growth rate $r$. The centre case (black curve, identical in all plots) has parameters $p=1$, $k=1, m=2, c=4, r=1$. In each plot, the varied parameter takes logarithmically spaced values from 0.4 to 2.5 of its centre-case value (rainbow curves, red to violet). All trajectories have initial condition $(b(0), h(0))=(1,0.1)$. Note that the ordinate scale differs between panels.

can be defined as

$U=\frac{k m}{c p}, \quad V=\frac{r m}{k}$

Equations (11) and (12) have two equilibrium points (denoted $\mathrm{A}$ and $\mathrm{B})$, given by:

Point A: $\quad b^{Q A}=p / k, \quad h^{Q A}=0$

Point B: $\quad b^{Q B}=m / c, \quad h^{Q B}=(p / m)-(k / c)$

These points have the following properties.

1. Point $\mathrm{A}$, the biosphere-only equilibrium, occurs in the absence of humans $(h=0)$, when the biosphere equilibrates to a biomass $b^{Q A}=p / k$ at which production $(p)$ balances respiration $(k b)$. Point $\mathrm{A}$ is a saddle point with its stable axis along the line $h=0$ (Appendix A).

2. As soon as $h$ exceeds zero for any reason, the system leaves point $\mathrm{A}$ and approaches point $\mathrm{B}$, the equilibrium for coexistence of a human population with the biosphere. Point B is always a stable equilibrium point (Appendix A). It is a stable focus (spiral trajectories) when $V>(4 U(1-U))^{-1}$, and a stable node (non-spiral trajectories) otherwise.

3. Production $(p)$ determines the equilibrium biomass at point $\mathrm{A}\left(b^{Q A}\right)$, but at point $\mathrm{B}, p$ instead determines the equilibrium human population $\left(h^{Q B}\right)$. The biomass at point $\mathrm{B}\left(b^{Q B}\right)$ is independent of $p$ and is determined by $m$ and $c$, attributes of the human population.
4. Points A and B are both independent of the growth rate $r$ and therefore of the group $V$. The role of $r$ (and $V$ ) is to determine the nature of the approach to point $\mathrm{B}$, as illustrated below.

5. For $h^{Q B}$ to be positive, the parameters must satisfy $0<U<1$.

A "resource condition index" $W$ can be defined as the ratio of the equilibrium biomass values with and without human utilisation:

$W=\frac{b^{Q B}}{b^{Q A}}$

In the presence of a human population at equilibrium, a fraction $W$ of the potential (unutilised) biomass remains in place, and a fraction $(1-W)$ is removed by utilisation. Equations (13) and (14) show that for the basic system governed by Eqs. (11) and (12), we have $W=U$. (In an extended version of this model considered below, $W$ is a function of $U$ ). The fractional human appropriation of net primary production, or HANPP (Boyden, 2004), is $g_{2} / g_{1}=c b h / p$, which for the basic model at equilibrium point $\mathrm{B}$ is $1-U=1-W$.

Figure 1 illustrates the system dynamics by plotting trajectories $(b(t), h(t))$ on the $b h$ plane under four scenarios, respectively corresponding to variation of $p, m, c$ and $r$ about a centre case with $p=1, k=1, m=2, c=4, r=1$. The total range for the varied parameter is about a factor of 5 in each case. The initial condition is that the biomass takes the potential value $b^{Q A}$ (= 1 with the centre-case parameter choices) with a small human population.

- Scenario 1 (variation of $p$ ): As $p$ (the primary production of biomass) increases, the system responds through an increase in the equilibrium human population $\left(h^{Q B}=p / m-k / c\right)$, not the equilibrium biomass $\left(b^{Q B}=m / c\right)$, as noted above. For low values of $p$, the dimensionless group $U$ exceeds 1 and the coexistence equilibrium (point $B$ ) is no longer viable as it is both unphysical $\left(h^{Q B}<0\right)$ and also unstable, so the system reverts to the biosphere-only equilibrium (point $\mathrm{A}$ ). This occurs at different points along the $b$ axis under variation of $p$, since $b^{Q A}=p / k$.

- Scenario 2 (variation of $m$ ): One might expect that decreasing the human maintenance requirement $m$ would cause the human population to "walk more lightly upon the land", increasing the equilibrium resource condition index $W$. However, the reverse is the case: decreasing $m$ decreases $W$, increases the equilibrium human population rapidly, and decreases the equilibrium biomass. With decreasing $m$ there is a decreasing tendency of trajectories to spiral, and low $m$ values are associated with nodes (non-spiral trajectories near equilibrium point B).

- Scenario 3 (variation of c): This corresponds to variation of the rate of extraction of biomass by humans, or 
the intensity of human exploitation of the biosphere. Increasing $c$ causes the equilibrium biomass to decrease (as might be intuitively expected) but the human population increases only slowly. Also, as $c$ increases, there is an increase in the amplitude of oscillations associated with spiral orbits. The qualitative insight provided by this scenario is that more aggressive resource extraction has the counter-intuitive effect of decreasing the equilibrium biomass while not increasing the equilibrium human population by anything like as much. Equation (14) shows that as $c \rightarrow \infty, h^{Q B}$ approaches the upper-limit value of $p / m$ while $b^{Q B}$ approaches zero. In this limit the biomass is over-exploited without a return in the form of a high human population as in Scenario 2.

- Scenario 4 (variation of $r$ ): Under variation of the growth rate of the human population per unit biomass surplus, equilibrium point B does not change (see property 4 , above) but there is an increase in the amplitude of the decaying oscillations with which the system approaches this point. Hence, increase of $r$ increases the tendency of the system to exhibit "boom-bust" oscillations. A similar trend is evident with increasing $c$, although in that case there is also a shift in the equilibrium point as noted in the previous paragraph.

The oscillatory behaviour of this simple model (especially at high $c$ and $r$ values) echoes the hypothesis of Flannery (1994) that when humans move into a previously unoccupied ecosystem, the biosphere-human system undergoes an initial rapid exploitation phase, a resource crash accompanied by rapid decrease in the human population, and finally an equilibration.

\section{Biosphere-human interactions: extended model}

\subsection{Model formulation}

The above basic two-equation model of biosphere-human interactions is open to several criticisms (other than those associated with the extreme idealisation to just two state variables). Two of the main ones are: (1) the primary production $p$ is assumed to be constant at all levels of the biomass $b$, whereas production is actually limited (approximately linearly) by $b$ at low $b$, and saturates to a constant value at high $b$; and (2) the harvest flux $c b h$ is assumed in the basic model to be resource $(b)$ limited at all resource levels, so there is no resource level (no matter how large) at which the harvest flux saturates with respect to $b$. To investigate the effect of these possible limitation and saturation attributes of the production and harvest fluxes, we extend the basic model from class P0U1 to class P2U2.
The extended model is

$$
\begin{aligned}
& \frac{d b}{d t}=p\left(\frac{b}{b+b_{P}}\right)-k b-c b h\left(\frac{b_{H}}{b+b_{H}}\right) \\
& \frac{d h}{d t}=r c b h\left(\frac{b_{H}}{b+b_{H}}\right)-r m h
\end{aligned}
$$

where $b_{P}$ and $b_{H}$ are respectively the biomass scales for resource saturation of production and harvest. The factors in brackets, accounting for resource saturation, are written in a form which keeps the dimensions of $p, c, k, m$ and $r$ the same as in the basic model. As $b_{P} \rightarrow 0$ and $b_{H} \rightarrow \infty$, these factors approach 1 and Eqs. (16) and (17) revert to Eqs. (11) and (12).

The model now has seven dimensional parameters $(p, c$, $\left.k, m, r, b_{P}, b_{H}\right)$ and three dimensions ([B], [H], [T]). Hence there are four independent dimensionless groups. With this many parameters, analysis is greatly helped by normalising the model rigorously to a dimensionless form. (This was not done in the foregoing analysis of the basic model; the dimensionless approach provides a more concise description at the expense of the need for careful interpretation when parameters appear in both dimensionless groups and scales, as illustrated below). Dimensionless versions of the model variables $b, h$ and $t$ are defined as $x_{1}=b / b_{\text {scale }}, x_{2}=h / h_{\text {scale }}$ and $s=t / t_{\text {scale }}$, where $b_{\text {scale }}, h_{\text {scale }}$ and $t_{\text {scale }}$ are scales to be constructed from the externally specifed parameters. They are chosen as follows: $b_{\text {scale }}$ is the equilibrium biomass in the absence of a human population, $b_{\text {scale }}=b^{Q A}=(p / k)-b_{P}$, so that $x_{1}=1$ for the equilibrium biosphere without utilisation; $h_{\text {scale }}$ is set as $h_{\text {scale }}=r b_{\text {scale }}$, because $r$ is the obvious parameter with dimension $\left[\mathrm{H} \mathrm{B}^{-1}\right]$ for relating the scales for $h$ and $b$; and $t_{\text {scale }}$ is chosen as $1 / k$, the intrinsic biospheric time scale. With these choices, the dimensionless biomass, human population and time are

$x_{1}=\frac{b}{p / k-b_{P}}, \quad x_{2}=\frac{b}{r\left(p / k-b_{P}\right)}, \quad s=k t$

The four independent dimensionless groups are chosen as

$$
U=\frac{m}{c b^{Q A}}, \quad V=\frac{r m}{k}, \quad a_{1}=\frac{b_{P}}{b^{Q A}}, \quad a_{2}=\frac{b^{Q A}}{b_{H}}
$$

The definition of $U$ reverts to that for the basic model (Eq. 13) as $b_{P} \rightarrow 0$, and the definition of $V$ is identical to that for the basic model. Substituting these dimensionless variables into Eqs. (16) and (17), the dimensionless form of the extended model is found to be:

$$
\begin{aligned}
& \frac{d x_{1}}{d s}=\frac{\left(1+a_{1}\right) x_{1}}{x_{1}+a_{1}}-x_{1}-\frac{V x_{1} x_{2}}{U\left(1+a_{2} x_{1}\right)} \\
& \frac{d x_{2}}{d s}=\frac{V x_{1} x_{2}}{U\left(1+a_{2} x_{1}\right)}-V x_{2}
\end{aligned}
$$

The basic model (without resource saturation of production and harvest) is recovered as $b_{P} \rightarrow 0$ and $b_{H} \rightarrow \infty$, or as $a_{1} \rightarrow 0$ 


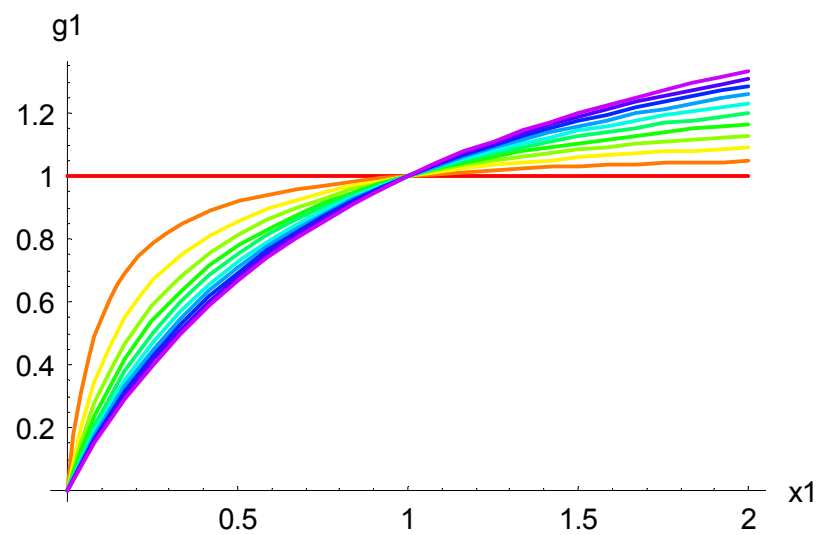

Fig. 2. Production term in the dimensionless extended biospherehuman model, $g_{1}\left(x_{1}\right)=\left(1+a_{1}\right) x_{1} /\left(x_{1}+a_{1}\right)$, plotted against $x_{1}$ for $a_{1}$ ranging from 0 to 1 .
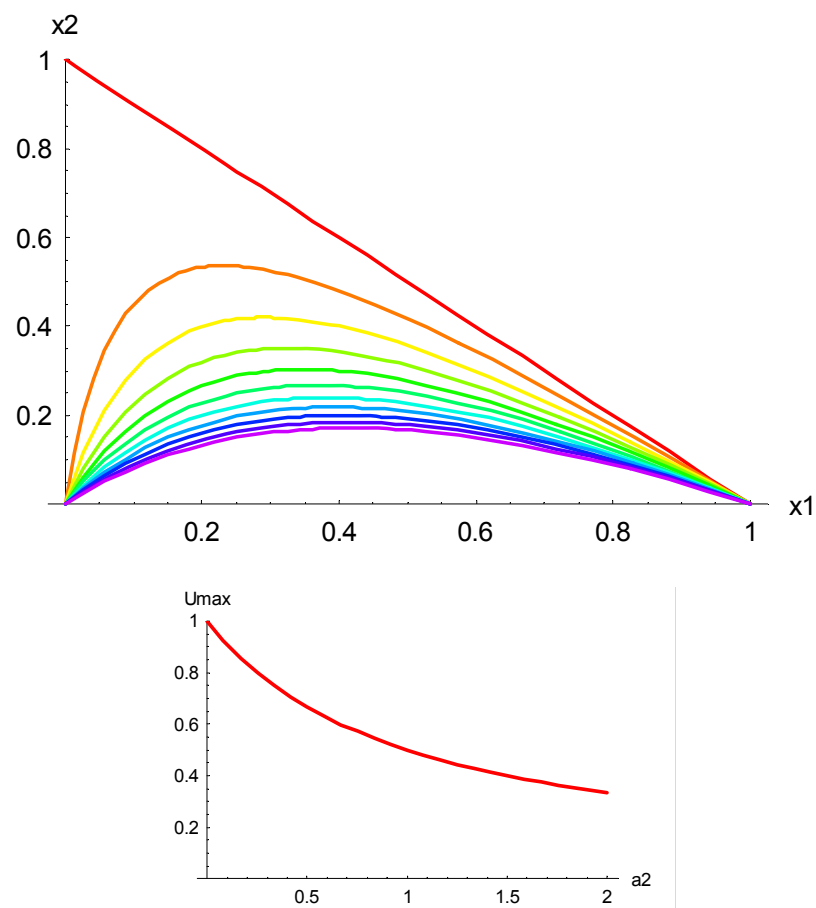

Fig. 3. (top) Coexistence equilibrium $\left(x_{1}^{Q B}, x_{2}^{Q B}\right)$ for the dimensionless extended biosphere-human model with $V=1$, plotted on the $x_{1} x_{2}$ plane with $W$ varying parametrically from 0 to 1 along each curve (left to right) and $a_{1}$ varying from 0 to 1 across curves (red: $a_{1}=0$; violet; $a_{1}=1$ ). (bottom) $U_{\max }=1 /\left(1+a_{2}\right)$ as a function of $a_{2}$.

and $a_{2} \rightarrow 0$. The reason for defining $a_{2}$ as proportional to $1 / b_{H}$ rather than $b_{H}$ is that it is more convenient to take the zero than the infinite limit in computations.

The production term in Eq. (20), $g_{1}\left(x_{1}\right)=$ $\left(1+a_{1}\right) x_{1} /\left(x_{1}+a_{1}\right)$, is plotted against $x_{1}$ in Fig. 2 for a range of $a_{1}$ values. The choice $a_{1}=0$ gives constant produc- tion $\left(g_{1}=1\right)$, while all other choices give a resource-limited production with $g_{1}=0$ at $x_{1}=0$ and $g_{1}=1$ at $x_{1}=1$.

\subsection{Equilibrium points}

Equations (20) and (21) have three equilibrium points at which $d x_{1} / d s=0$ and $d x_{2} / d s=0$ :

Point Z: $\quad\left(x_{1}^{Q Z}, x_{2}^{Q Z}\right)=(0,0)$

Point A: $\quad\left(x_{1}^{Q A}, x_{2}^{Q A}\right)=(1,0)$

Point B: $\left\{\begin{aligned} x_{1}^{Q B} & =\frac{U}{1-a_{2} U} \\ x_{2}^{Q B} & =\frac{U\left(1-a_{2} U-U\right)}{V\left(1-a_{2} U\right)\left(a_{1}\left(1-a_{2} U\right)+U\right)}\end{aligned}\right.$

Points A and B are respectively a biosphere-only equilibrium and a biosphere-human coexistence equilibrium, similar to those for the basic model (Eq. 14). Point $\mathrm{Z}$ is an additional equilibrium point at the origin, with biomass and human population both zero. Evaluation of the resource condition index $W$, defined by Eq. (15), gives

$W=\frac{x_{1}^{Q B}}{x_{1}^{Q A}}=\frac{U}{1-a_{2} U}, \quad U=\frac{W}{1+a_{2} W}$

Hence, for the extended model, $W$ is a function of the dimensionless group $U$, in contrast with the basic model for which $W=U$. Substituting $W$ for $U$ in Eq. (22), equilibrium point $\mathrm{B}$ can be written in the alternative, simpler form

$x_{1}^{Q B}=W, \quad x_{2}^{Q B}=\frac{W(1-W)}{V\left(a_{1}+W\right)}$

Biophysically realistic equilibrium solutions can only exist in a subset of parameter space. First, all parameters must be non-negative. Second, for the biosphere-only equilibrium biomass $\left(b^{Q A}\right)$ to be positive, it is necessary that $b_{\text {scale }}>0$, which requires that $(p / k)>b_{P}$. This is a condition on the dimensional parameters which becomes implicit when the model is made dimensionless, being incorporated into a requirement on $b_{\text {scale }}$. Third, the equilibrium biomass in a harvested system cannot exceed the equilibrium biomass without harvest, so biophysically realistic solutions at equilibrium point B exist only when $W$ is between 0 and 1. From Eq. (23), this means that $0<U<U_{\max }$, where $U_{\max }=1 /\left(1+a_{2}\right)$. This is the counterpart for the extended model of the requirement $0<U<1$ for the basic model.

Figure 3 shows the behaviour of equilibrium point $\mathrm{B}$ on the $x_{1} x_{2}$ plane in response to variation of the parameters $a_{1}$ (which varies 0 to 1 across curves) and $U$ (which varies parametrically along each curve from 0 to $\left.U_{\max }\right)$. This variation of $U$ means that $W$ varies from 0 to 1 along each curve. The curves do not change as $a_{2}$ is varied, but the parametrically varying $U$ values along each curve change with $a_{2}$ because of the dependence of $U_{\max }$ on $a_{2}$, shown in the small lower panel of Fig. 3. The main panel of Fig. 3 assumes $V=1$, the effect of increasing (decreasing) $V$ being 

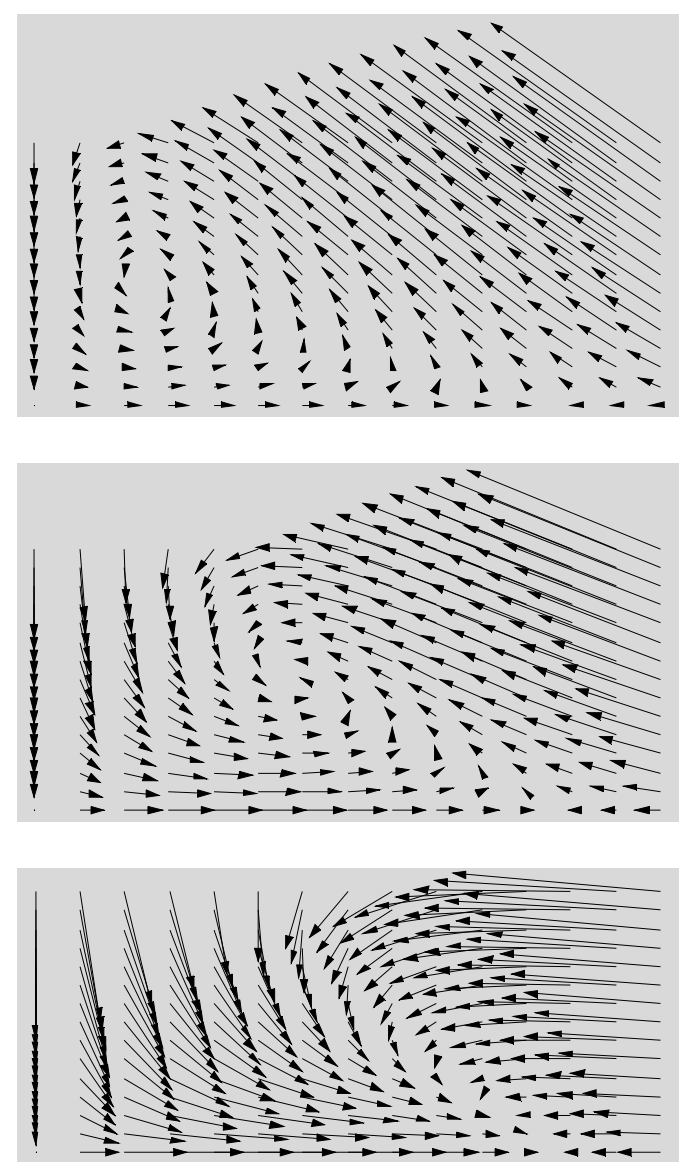

Fig. 4. Flow fields on $x_{1} x_{2}$ plane for the dimensionless extended biosphere-human model, with $V=1, a_{1}=a_{2}=0.5$, and $W=0.2$ (top), 0.5 (middle) and 1.0 (bottom). The $x_{1}$ (horizontal) axis extends from 0 to 1.2 , and the $x_{2}$ (vertical) axis from 0 to 0.5 .

to shrink (stretch) the vertical axis. The most important aspect of this figure is the change in the behaviour of equilibrium point $B$ in the transition from the basic model (with constant production and $a_{1}=0$ ) to the extended model (with biomass-limited production and $\left.a_{1}>0\right)$. As resource condition declines ( $W \rightarrow 0$ or $U \rightarrow 0$ ), the human population in the basic model increases $\left(x_{2}^{Q B} \rightarrow 1 / V, h^{Q B} \rightarrow(k / m) b^{Q B}\right)$, but in the model with biomass-limited production, $x_{2}^{Q B}$ and $h^{Q B}$ both decline (more realistically) to zero.

\subsection{Trajectories}

A first glimpse into the dynamical behaviour of the extended model is provided in Fig. 4, in which the flow vector $\left(f_{1}\left(x_{1}, x_{2}\right), f_{2}\left(x_{1}, x_{2}\right)\right)=\left(d x_{1} / d s, d x_{2} / d s\right)$ is plotted on the $x_{1} x_{2}$ plane for three different $W$ values, $0.2,0.5$ and 1 (other parameters are $V=1, a_{1}=0.5, a_{2}=0.5$ ). For $W=0.2$ and 0.5 , the oscillatory nature of the flow around equilibrium point $\mathrm{B}$ is clear. For $W=1$, point $\mathrm{B}$ coincides with point $\mathrm{A}$, the biosphere-only equilibrium.
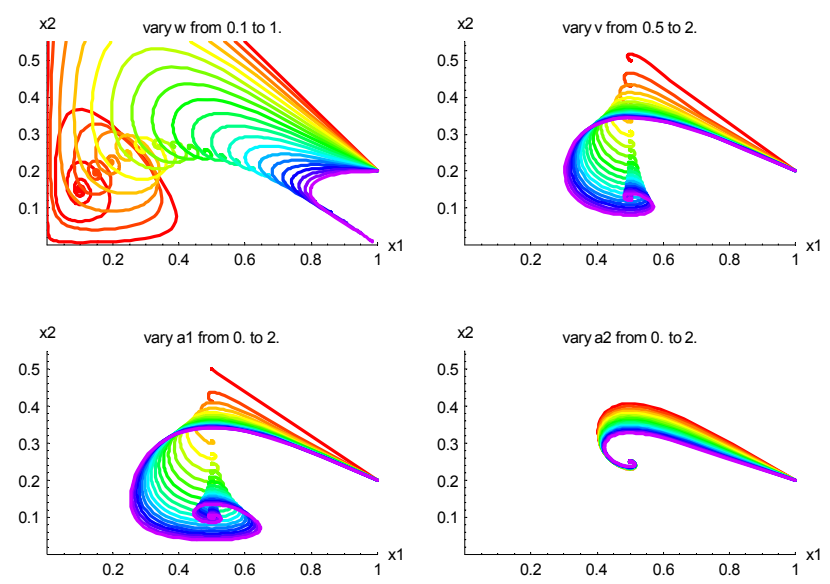

Fig. 5. Trajectories on $x_{1} x_{2}$ plane for the dimensionless extended biosphere-human model, with centre-case parameters $W=0.5$, $V=1, a_{1}=0.5, a_{2}=0.5$. The initial condition is always $x_{1}=1.0$, $x_{2}=0.2$. Panels show (with colours proceeding through the rainbow from red to violet) the effect of (a) variation of $W$ from 0.1 to 1; (b) variation of $V$ from 0.5 to 2 ; (c) variation of $a_{1}$ from 0 to 2 ; (d) variation of $a_{2}$ from 0 to 2 .

Figure 5 shows the response of trajectories to variation (in turn) of $W, V, a_{1}$ and $a_{2}$ around the centre case $W=0.5$, $V=1, a_{1}=0.5$ and $a_{2}=0.5$. This is a high-level summary of the response of the system to changes in external conditions, but it needs care in interpretation because dimensional parameters $\left(p, c, k, m, r, b_{P}, b_{H}\right)$ affect both the dimensionless groups ( $W$ or $U, V, a_{1}$ and $a_{2}$ ) and also the normalising scales $\left(b_{\text {scale }}, h_{\text {scale }}\right.$ and $\left.t_{\text {scale }}\right)$. To infer the response of dimensional state variables $(b$ and $h)$ to changes in dimensional external parameters with Fig. 5 and similar dimensionless plots, it is necessary to consider the influences of the dimensional parameters both on the dimensionless groups and also on the scales with which the axes in Fig. 5 are normalised. Keeping this in mind, the implications of Fig. 5 are as follows.

- Variation of $W$ : Since $W$ is a function of $U$ through Eq. (23), variation of $W$ from 0 to 1 occurs as $U$ varies from 0 to $U_{\max }$. As this occurs, the equilibrium point follows a trajectory consistent with Fig. 3. The rate of convergence to equilibrium (the rate at which the amplitude of successive spirals diminishes) increases with $W$ and $U$, so that the system is more prone to strong oscillatory behaviour at low than at high $W$. Since $U$ is defined in terms of dimensional parameters by $U=m /\left(c b^{Q A}\right)=m /\left(c\left((p / k)-b_{P}\right)\right)$, variation of $U$ (and $W)$ can occur through variation of any of $p, m, c, k$ or $b_{P}$. Hence this variation is the counterpart for the extended model of all of scenarios 1, 2 and 3 for the basic model. Variation of $p, k$ and $b_{P}$ also affects the equilibrium biomass scale $b^{Q A}=p / k-b_{P}$, but this affects the scaling on both the $x_{1}$ and $x_{2}$ axes in a similar way. 


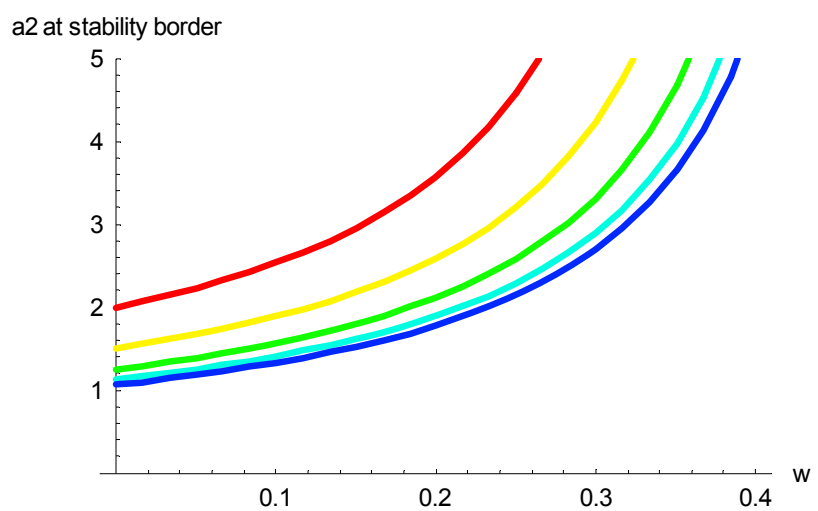

Fig. 6. Instability threshold for the coexistence equilibrium point of the dimensionless extended biosphere-human model as a function of $a_{1}, a_{2}$ and $W$. Curves show the instability threshold on the ( $\left.W, a_{2}\right)$ plane, with $a_{1}=1,2,4,8,16$ (red to blue). Points above the curves are unstable.
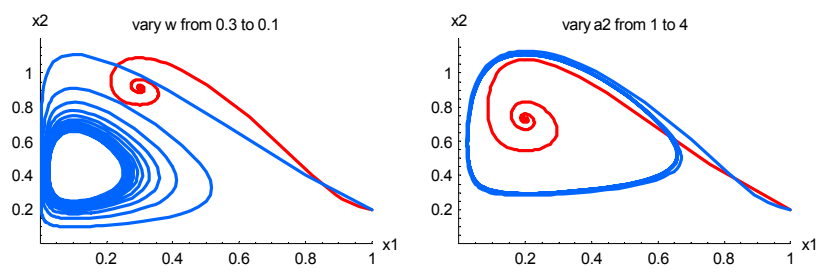

Fig. 7. Trajectories on $x_{1} x_{2}$ plane for the dimensionless extended biosphere-human model illustrating the effect of crossing the instability threshold for the coexistence equilibrium (point B). Centrecase parameters are $W=0.2, V=0.1, a_{1}=2, a_{2}=2$. Left panel: two trajectories with $W=0.3$ and 0.1 and other parameters at centre-case values. Right panel: two trajectories with $a_{2}=1$ and 4 , and other parameters at centre-case values. In each case the first (red) trajectory is stable, and the second (blue) trajectory is unstable, entering a limit cycle.

- Variation of $V$ : This variation reflects essentially a variation in the growth rate $r$. As $V$ increases the oscillatory tendency of the model increases, as for the basic model (Fig. 1). With increasing $V$ there is also a decrease in $x_{2}^{Q B}$, the equilibrium dimensionless $h^{Q B}$, whereas the equilibrium point $\left(b^{Q B}, h^{Q B}\right)$ for the basic model is independent of $r$ and $V$ (see Scenario 4 for the basic model). The apparent difference arises because $r$ appears in the normalisation of $h^{Q B}$ to $x_{2}^{Q B}$ (see Eq. 18).

- Variation of $a_{1}$ :Increasing $a_{1}$ occurs with increase of $b_{P}$ and thus the limitation of production at low biomass and saturation at high biomass (Fig. 2). This has a strong tendency to increase the oscillatory behaviour of the model, and also causes a reduction in $x_{2}^{Q B}$, the equilibrium dimensionless $h^{Q B}$, while $x_{1}^{Q B}$ stays constant (a trend also evident in Fig. 3).
- Variation of $a_{2}$ : Increasing $a_{2}$ occurs with progressively more saturation of the harvest flux at high biomass, and with decreasing $b_{H}$. For the parameter range shown in Fig. 5, increase in $a_{2}$ causes a mild decrease in the oscillatory tendency of the trajectories while leaving the equilibrium point $\left(x_{1}^{Q B}, x_{1}^{Q B}\right)$ unchanged.

\subsection{Stability}

The stability of the equilibrium points for the extended model is more subtle than for the basic model, for which the coexistence equilibrium (point B) is stable for all parameter choices. Stability analysis for the extended model leads to the following conclusions (see details in Appendix B).

- Equilibrium point $Z$ (the origin) is a saddle point with its stable axis oriented along the $x_{2}$ axis, so point $\mathrm{Z}$ is unstable with respect to an infinitesimal variation in $x_{1}$ and stable with respect to a variation in $x_{2}$. Hence a small positive perturbation in biomass from point $\mathrm{Z}$ causes the biosphere to move away from point $\mathrm{Z}$ and approach point $\mathrm{A}$, whereas a small human population dies out as it has nothing to live on.

- Point A (the biosphere-only equilibrium) is a saddle point with its stable axis in the $x_{1}$ direction, as in the basic model. In the absence of humans, the biosphere approaches point $\mathrm{A}$ along the $x_{1}$ axis from either direction. A small positive perturbation in $h$ or $x_{2}$ causes the system to leave point $\mathrm{A}$ and approach point $\mathrm{B}$.

- Point B (the coexistence equilibrium) can be either stable or unstable, depending on values of $W, a_{1}$ and $a_{2}$.

The condition for stability of point B is (see Appendix B):

$Y>0 \quad$ (stable)

$Y<0 \quad$ (unstable)

with $\quad Y=1+a_{1}-a_{1} a_{2}+2 a_{1} a_{2} W+a_{2} W^{2}$

Hence, for given $W$ and $a_{1}$, instability occurs when $a_{2}$ exceeds a threshold value:

$a_{2}>a_{2 \text { Thresh }}=\frac{1+a_{1}}{a_{1}-2 a_{1} W-W^{2}}$

This threshold value is plotted on the $\left(W, a_{2}\right)$ plane in Fig. 6 , for several values of $a_{1}$. Instability occurs when $W$ is low and $a_{2}$ and $a_{1}$ are high. Figure 7 shows how the system behaviour changes as $W$ and $a_{2}$ cross this threshold. When the parameters are on the stable side of the threshold, trajectories are attracted to point $\mathrm{B}$, but for parameters on the unstable side of the threshold, trajectories are repelled from point B and enter a limit cycle in which oscillatory behaviour of the system does not die away but continues for all time.

To conclude the analysis of the simple model of biospherehuman interactions, we summarise four significant differences between its basic (constant-production) and extended 
(resource-limited production and utilisation) forms. First, the basic model has biosphere-only and coexistence equilibrium points, but the extended model has an additional equilibrium point at the origin. Second, with declining resource condition $(W), h$ increases in the basic model but (more realistically) declines toward zero in the extended model. Third, the extended model is more prone to strong oscillatory behaviour than the basic model, especially at low $W$. Fourth, in the basic model the coexistence equilibrium is always stable, but in the extended model it becomes unstable at low $W$ and with strong resource limitation of production and/or utilisation (large $a_{1}$ or $a_{2}$ ). In these conditions, trajectories enter a limit cycle of orbits about the coexistence equilibrium point, rather than eventually reaching it.

\section{Plant and soil carbon dynamics}

Carbon dynamics in the plant-soil system provides an example of a producer-utiliser system which operates in processor mode, as defined in the introduction. The producers are plants, through the assimilation of atmospheric $\mathrm{CO}_{2}$ into biomass, and the utilisers are soil heterotrophic organisms which feed off plant litter and respire the carbon back to the atmosphere as $\mathrm{CO}_{2}$.

\subsection{Model formulation}

The system is modelled using an idealised, two-equation representation with state variables for the stores of biomass carbon $\left(x_{1}\right)$ and litter and soil carbon $\left(x_{2}\right)$. The governing equations are:

$$
\begin{aligned}
\frac{d x_{1}}{d t} & =F(t)\left(\frac{x_{1}}{x_{1}+q_{1}}\right)\left(\frac{x_{2}}{x_{2}+q_{2}}\right)+s_{1}-k_{1} x_{1} \\
\frac{d x_{2}}{d t} & =k_{1} x_{1}-k_{2} x_{2}
\end{aligned}
$$

where $F(t)$ is a forcing term describing the net primary production (NPP); $q_{1}$ and $q_{2}$ are scales for the limitation of production by lack of $x_{1}$ and $x_{2}$, respectively; $k_{1}$ and $k_{2}$ are rate constants for the decay of $x_{1}$ and $x_{2}$, respectively; and $s_{1}$ is a component of the primary production which is independent of both $x_{1}$ and $x_{2}$. We consider both the case where $F(t)$ is independent of time, $F(t)=F_{0}$, and also the case where $F(t)$ is a random function of time. The model parameters are $q_{1}$, $q_{2}, k_{1}, k_{2}$, and $s_{1}$, together with $F_{0}$ or parameters characterising $F(t)$ as a random function.

These equations are a simplification the carbon dynamics in typical terrestrial biosphere models, including models of global vegetation dynamics as in the DGVM intercomparison of Cramer et al. (2001), and the terrestrial biosphere components of coupled carbon-climate models as in the $\mathrm{C}^{4} \mathrm{MIP}$ intercomparison of Friedlingstein et al. (2006). Characteristics of such terrestrial biosphere models at several levels of complexity are reviewed by Raupach et al. (2005). Relative to sophisticated terrestrial biosphere models, Eqs. (27) and (28) are an extreme idealisation: all biomass carbon (leaf, wood, root) is lumped into a single store $x_{1}$ governed by an equation of the form $d x_{1} / d t=(\mathrm{NPP})-$ (litterfall), and all litter and soil carbon into a single store $x_{2}$ governed by $d x_{2} / d t=($ litterfall)-(heterotrophic respiration). Litterfall and heterotrophic respiration are parameterised as first-order decay fluxes, $k_{1} x_{1}$ and $k_{2} x_{2}$. NPP is assumed to depend on three factors: (1) a forcing term $F(t)$ representing the fluctuating availability of light and water resources through weather and climate variability, (2) a factor $x_{1} /\left(x_{1}+q_{1}\right)$ of Michaelis-Menten form describing the limitation of NPP by lack of biomass in resource-gathering organs (leaves, roots), and (3) a factor $x_{2} /\left(x_{2}+q_{2}\right)$, also of Michaelis-Menten form, describing the integrated symbiotic effects of soil carbon on plant productivity. This symbiotic factor accounts for the overall beneficial effect of soil carbon on plant growth, through processes such as nutrient cycling and improvement in soil water holding capacity (often not included in sophisticated terrestrial biosphere models such as those surveyed in the references above). The parameter $s_{1}$ represents a (small) production term that is not dependent on $x_{1}$ and $x_{2}$, for example generation of biomass from a long-term reservoir of seed propagules. For the present purpose, $s_{1}$ is assumed to be a constant flux independent of external conditions as well as $x_{1}$ and $x_{2}$.

Equations (27) and (28) are identical to the test model used in the OptIC (Optimisation Intercomparison) project (Trudinger et al., 2007; also http://www.globalcarbonproject. org). The aim of OptIC is to compare several modeldata synthesis (parameter estimation and data assimilation) approaches for determining parameters in biogeochemical models from multiple sources of noisy data. Equations (27) and (28) are used as a simple test model which embodies features of a real biogeochemical model, together with generated data from model forward runs with added noise, for which "true" parameters are known.

\subsection{Equilibrium points and stability}

We consider first the situation with steady forcing, $F(t)=F_{0}$. Seeking the equilibrium points points $\mathbf{x}^{Q}$ at which $d x_{1} / d t=$ $d x_{2} / d t=0$, Eq. (28) shows that

$x_{2}^{Q}=x_{1}^{Q} k_{1} / k_{2}$

and Eq. (27) implies that $x_{1}^{Q}$ satisfies the cubic equation

$$
\begin{aligned}
& j\left(x_{1}\right)=c_{0}+c_{1} x_{1}+c_{2} x_{1}^{2}+c_{3} x_{1}^{3}=0 \\
& \left(\begin{array}{l}
c_{0} \\
c_{1} \\
c_{2} \\
c_{3}
\end{array}\right)=\left(\begin{array}{c}
q_{1} q_{2} k_{2} s_{1} / k_{1}^{2} \\
\left(\left(q_{1} k_{1}+q_{2} k_{2}\right) s_{1}-q_{1} q_{2} k_{1} k_{2}\right) / k_{1}^{2} \\
\left(F_{0}-q_{1} k_{1}-q_{2} k_{2}+s_{1}\right) / k_{1} \\
-1
\end{array}\right)
\end{aligned}
$$




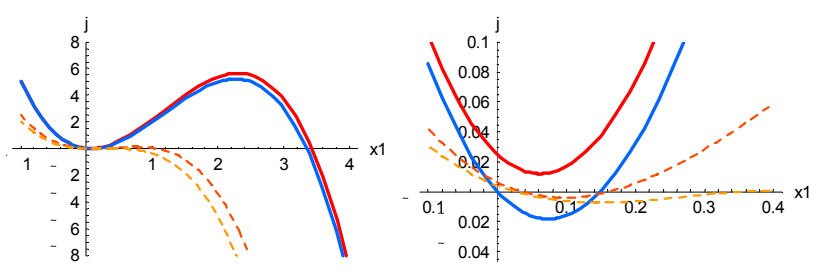

Fig. 8. The cubic $j\left(x_{1}\right)$ defined by Eq. (30), with reference-case parameter values $F_{0}=1, q_{1}=1, q_{2}=1, k_{1}=0.2, k_{2}=0.1, s_{1}=0.01$. Solid red and blue lines show the effect of varying $s_{1}$ (red: $s_{1}=0.01$; blue: $s_{1}=0$, with other parameters at reference-case values). Dashed orange (heavy) and yellow (light) lines show effect of varying $k_{1}$ (orange: $k_{1}=0.4$; yellow: $k_{1}=0.5$, with other parameters at reference-case values). Left panel shows all zeros of $j\left(x_{1}\right)$. Right panel is an expanded view showing $j\left(x_{1}\right)$ near the origin.

Thus the equilibrium points are of the form $\mathbf{x}^{Q}=\left(x_{1}^{Q}, x_{1}^{Q} k_{1} / k_{2}\right)$, where $x_{1}^{Q}$ is a solution of the cubic equation $j\left(x_{1}^{Q}\right)=0$. This equation has either one or three real roots, yielding either one or three equilibrium points. At least one root must be positive $\left(x_{1}^{Q}>0\right)$ for a nontrivial, biophysically meaningful solution to exist. The cubic $j\left(x_{1}\right)$ is plotted in Fig. 8 with reference-case parameters $F_{0}=1$, $q_{1}=1, q_{2}=1, k_{1}=0.2, k_{2}=0.1$ (the red curve; other curves are described below).

When the equilibrium points are determined by the roots of a single equation, it is not necessary to appeal to the Jacobian and its characteristic equation to determine stability. A sufficient criterion is that an equilibrium point $x_{1}^{Q}$ is stable if $d j / d x_{1}<0$ at $x_{1}=x_{1}^{Q}$, and unstable otherwise. Since $j\left(x_{1}\right)=-x_{1}^{3}+\ldots$, it is clear from the geometry (see Fig. 8) that if there is just one equilibrium point then it is stable, whereas if there are three equilibrium points, say $\mathrm{A}, \mathrm{B}, \mathrm{C}$ with equilibrium $x_{1}$ values $x_{1}^{Q A}, x_{1}^{Q B}$ and $x_{1}^{Q C}$ in increasing order, then $x_{1}^{Q A}$ and $x_{1}^{Q C}$ are stable and $x_{1}^{Q B}$ is unstable. For all biophysically admissible parameter choices, $j\left(x_{1}\right)$ has at least one stable root with $x_{1}>0$. This will be designated as $x_{1}^{Q C}$, the largest stable equilibrium value of $x_{1}$, and can be identified as a "healthy" or "active" equilibrium state of the system. It is important to understand whether and when there is another biophysically attainable and stable equilibrium state, equilibrium point $\mathrm{A}$, with $x_{1}^{Q A} \geq 0$. This depends on the parameter choices, particularly for $s_{1}$. There are three main possible kinds of behaviour, as follows.

1. If $s_{1}>0$ and the cubic $j\left(x_{1}\right)$ crosses the $x_{1}$ axis only once, then there is only one equilibrium point $x_{1}^{Q C}$, the "active-biosphere" point. It is always stable, so the system must approach it under steady forcing. This is the outcome with the reference-case parameters, as shown by the red curve in Fig. 8.

2. If $s_{1}>0$ and $j\left(x_{1}\right)$ crosses the $x_{1}$ axis three times, all greater than zero, then there are two stable, positive equilibrium points $\left(x_{1}^{Q A}\right.$ and $\left.x_{1}^{Q C}\right)$ on either side of one unstable point $\left(x_{1}^{Q B}\right)$. The two dashed curves in Fig. 8 show this outcome occurring as $k_{1}$ is increased from 0.2 to (respectively) 0.4 and 0.5 , with other parameters held at reference-case values. In this case, $x_{1}^{Q C}$ is the "active-biosphere" point as before, and $x_{1}^{Q A}$ is a "dormant-biosphere" equilibrium.

3. If $s_{1}=0$, then there is a stable equilibrium point (A) of Eqs. (27) and (28) at the origin, in addition to the "active-biosphere" equilibrium point $x_{1}^{Q C}>0$. (Existence of this root is assured because $s_{1}=0$ implies $c_{0}=0$, so $x_{1}^{Q}=0$ is a root of $j\left(x_{1}\right)$; stability follows because $d j / d x_{1}=c_{1}$ at $x_{1}=0$, and when $s_{1}=0$, we have $c_{1}=-q_{1} q_{2} k_{2} / k_{1}$, which is negative for positive values of $q_{1}, q_{2}, k_{2}$ ). The equilibrium point at the origin corresponds to "extinction" of the biosphere in this simple model system, since once the system reaches the origin with $s_{1}=0$, it remains there for all subsequent time, no matter what the forcing $F(t)$. The blue curve in Fig. 8 shows this case.

In addition to these three options, there are other possibilities. For some parameter combinations the cubic $j\left(x_{1}\right)$ has no positive or zero solutions (that is, all crossings of the $x_{1}$ axis occur when $x_{1}<0$ ), so these parameter combinations are not biophysically realisable. Also, if $s_{1}=0$ and either $q_{1}=0$ or $q_{2}=0$, the model relaxes to a simpler form as $j\left(x_{1}\right)$ is of lower degree than a cubic. In these cases there can be only one stable equilibrium point.

Figure 9 shows how the three main kinds of behaviour can all arise as parameters are varied around the reference case. Each panel of this figure superimposes plots of $x_{1}^{Q}$ (red), $x_{2}^{Q}$ (orange), Det $\mathbf{J}$ (green), - Tr $\mathbf{J}$ (blue) and the discriminant $\left(D=(\operatorname{Tr} \mathbf{J})^{2}-4\right.$ Det $\left.\mathbf{J}\right)$, at a particular equilibrium point (A, B, C, in different columns), and examines the response of these quantities to variation of a parameter $\left(s_{1}, k_{1}\right.$, $k_{2}, q_{1}, q_{2}$, in different rows). No lines are plotted where real equilibrium solutions do not exist. The trace and the determinant of $\mathbf{J}$ show the stability of the point, since Det $\mathbf{J}$ and $-\operatorname{Tr} \mathbf{J}$ must both be positive for stability, from Eq. (9). The discriminant shows whether local trajectories around the point are non-spiral or spiral, from Eq. (10). The picture is rich: the "active-biosphere" equilibrium point $\mathrm{C}$ exists as a stable node (non-spiral trajectories) for nearly all parameter choices. Points A and B form a pair, in that neither exists or both exist. When both exist, point $\mathrm{A}$ is always stable and point B always unstable. The discriminant is always positive where points exist, indicating that spiral behaviour is not observed in this model over the slices of parameter space surveyed in Fig. 9.

The non-spiral nature of the trajectories in this model is further illustrated in Fig. 10, where trajectories are plotted 

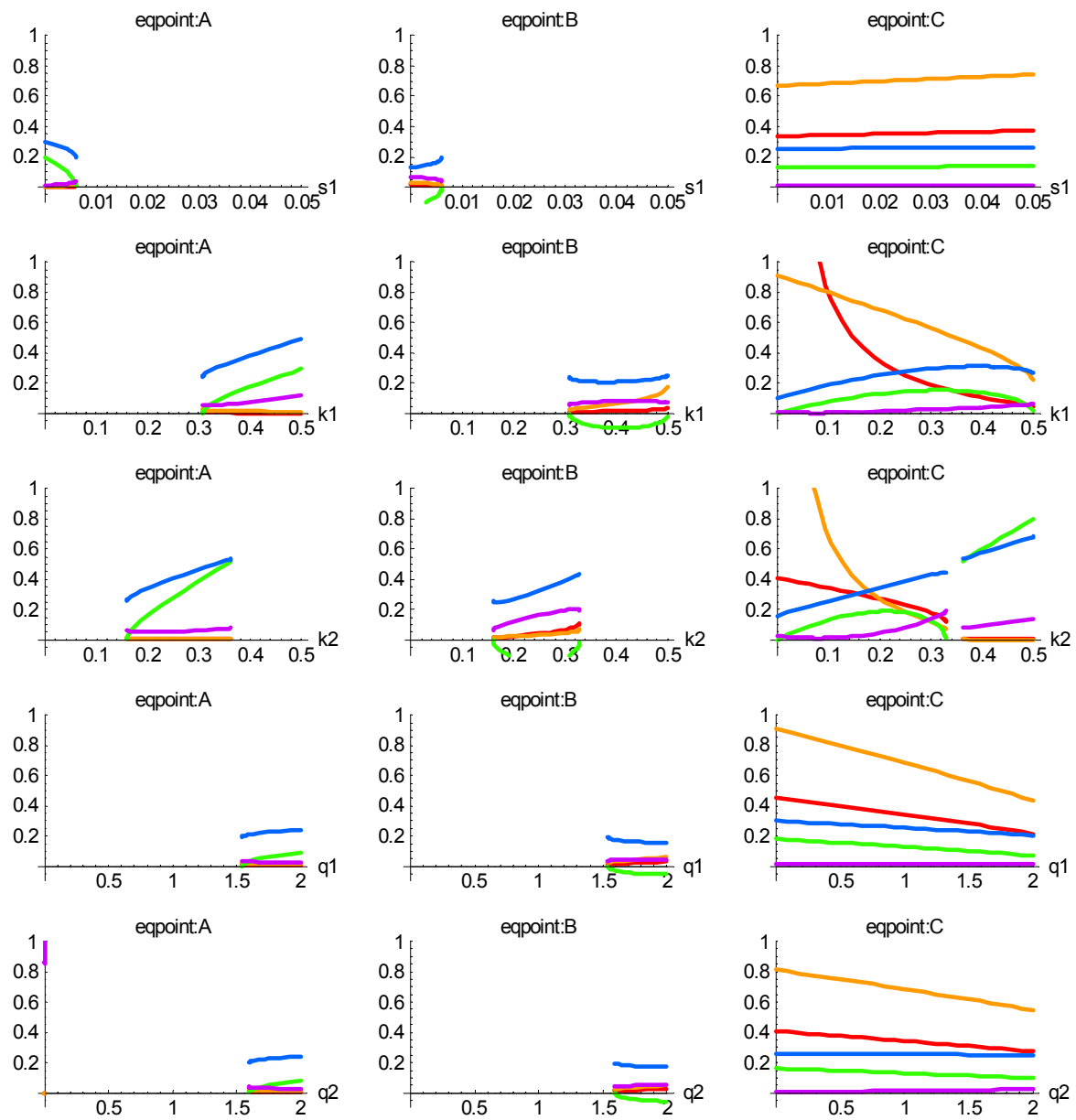

Fig. 9. Variation of $x_{1}^{Q} / 10$ (red), $x_{2}^{Q} / 10$ (orange), 10 Det $\mathbf{J}$ (green), - $\operatorname{Tr} \mathbf{J}$ (blue) and the discriminant $\left(D=(\operatorname{Tr} \mathbf{J})^{2}-4\right.$ Det $\left.\mathbf{J}\right)($ violet) at equilibrium points A, B, C (columns) for the two-equation model of plant $\left(x_{1}\right)$ and soil $\left(x_{2}\right)$ carbon dynamics, Eqs. (27) and (28), with steady forcing $\left(F(t)=F_{0}\right)$ and reference-case parameter values $F_{0}=1, q_{1}=1, q_{2}=1, k_{1}=0.2, k_{2}=0.1, s_{1}=0.01$. Rows 1 to 5 show effect of varying $s_{1}, k_{1}, k_{2}, q_{1}, q_{2}$ about reference-case values.

by numerically integrating Eqs. (27) and (28) for a number of parameter choices. In all cases the trajectories decay towards equilibrium, rather than spiralling towards it as for the biosphere-human model (Fig. 5). This is consistent with the behaviour of the discriminant (see Fig. 9 and Eq. (10)). An equivalent statement is that at all stable equilibrium points of the model, all eigenvalues of $\mathbf{J}$ are real and negative. This is in accord with the finding of Bolker et al. (1998) that the eigenvalues of the Century plant-soil carbon model are real and negative, so that the model shows no oscillatory behaviour.

\subsection{Random forcing}

To this point there has been no time-dependent forcing applied to any model considered. This section investigates the effect of random forcing $F(t)$, or "noise", on the system described by Eqs. (27) and (28). Random forcing here rep- resents the effects of fluctuating resource (water and light) availability on the net primary productivity of the system. When $F(t)$ is an externally prescribed random process, then the solutions $x_{1}(t)$ and $x_{2}(t)$ are also random processes.

The forcing $F(t)$ is prescribed here by taking its normalised logarithm $\left(\ln \left(F(t) / F_{0}\right)\right.$, where $F_{0}$ is a measure of the magnitude of $F(t)$ ) to be a Markovian, Gaussian random process $m(t)$ with zero mean, standard deviation $\sigma_{m}$ and time scale $T_{m}$. This process, known as the OrnsteinUhlenbeck process (van Kampen 1981), is fundamental in the theory of random processes; it has an exponential autocorrelation function $\left(\exp \left(-|\tau| / T_{m}\right)\right.$, where $\tau$ is the time lag) and a power spectrum with high-frequency roll-off proportional to (frequency) ${ }^{-2}$. In finite-difference form, at times $t_{i}$ with increments $\Delta t\left(<<T_{m}\right)$, the processes $m\left(t_{i}\right)$ and $F\left(t_{i}\right)$ obey

$m_{i}=\alpha m_{i-1}+\beta \sigma_{m} \zeta_{i} F\left(t_{i}\right)=F_{0} \exp \left(m_{i}\right)$ 

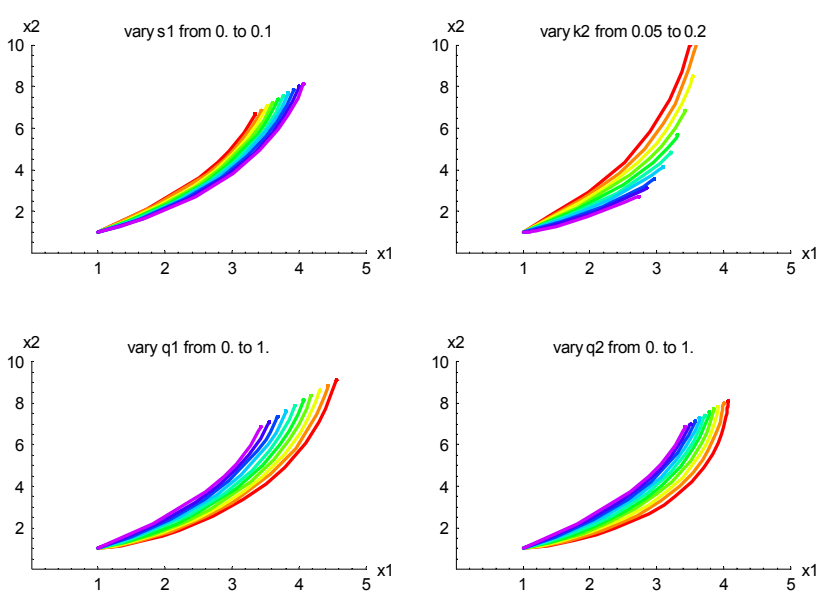

Fig. 10. Trajectories on $x_{1} x_{2}$ plane for the two-equation model of plant $\left(x_{1}\right)$ and soil $\left(x_{2}\right)$ carbon dynamics, Eqs. (27) and (28), with steady forcing $\left(F(t)=F_{0}\right)$ and reference-case parameter values $F_{0}=1, q_{1}=1, q_{2}=1, k_{1}=0.2, k_{2}=0.1, s_{1}=0.01$. The initial condition is always $x_{1}=1.0, x_{2}=1.0$. Panels show the effect of (a) variation of $s_{1}$ from 0 to 0.1 ; (b) variation of $k_{2}$ from 0.05 to 0.2 ; (c) variation of $q_{1}$ from 0 to 1 ; (d) variation of $q_{2}$ from 0 to 1. All these trajectories converge to the "active" equilibrium point, $\left(x_{1}^{Q C}, x_{2}^{Q C}\right)$.

where $\alpha=\exp \left(-\Delta t / T_{m}\right), \beta=\left(1-\alpha^{2}\right)^{1 / 2}$, and $\zeta_{i}$ is a Gaussian random number with zero mean and unit variance. This formulation ensures that $F\left(t_{i}\right)$ is always positive, with a mean determined by $F_{0}$ (in fact the mean of $F\left(t_{i}\right)$ is a little larger than $F_{0}$ because of nonlinearity). The parameters determining $F(t)$ are $F_{0}, \sigma_{m}$ and $T_{m}$ (but not $\Delta t$, which is merely a discretisation interval).

Figure 11 shows time series of $x_{1}(t)$ (red) and $x_{2}(t)$ (blue), calculated using a random forcing $F(t)$ with $F_{0}=1, \sigma_{m}=0.5$, $T_{m}=1$, and a computational time step $\Delta t=0.1$ time units. The forcing function $F(t)$ is shown in the bottom panel. In the top panel, the parameters are set at reference-case values (see figure caption). The behaviour of the system is (not surprisingly) that $x_{1}(t)$ and $x_{2}(t)$ fluctuate around the "activebiosphere" equilibrium point for the system with steady forcing, $\left(x_{1}^{Q C}, x_{2}^{Q C}\right)$. This is an example of the first kind of behaviour described above. For these parameter values there is only one stable equilibrium point $(\mathrm{C})$, so the system undergoes excursions around point $\mathrm{C}$ under random forcing.

The next two panels in Fig. 11 show the effects of increasing $k_{1}$ from its reference-case value of 0.2 to 0.4 and 0.5 , respectively. These parameter values illustrate the second kind of behaviour. There are now two stable equilibrium points, A and $\mathrm{C}$, with $\mathrm{C}$ being the "active-biosphere" point and $\mathrm{A}$ being a "dormant-biosphere" point close to, but not at, the origin. (The cubic curves $j\left(x_{1}\right)$ which determine equilibrium points $\mathrm{A}$ and $\mathrm{C}$ for these parameter values are shown as the dashed lines in Fig. 8). Under the influence of random forcing the
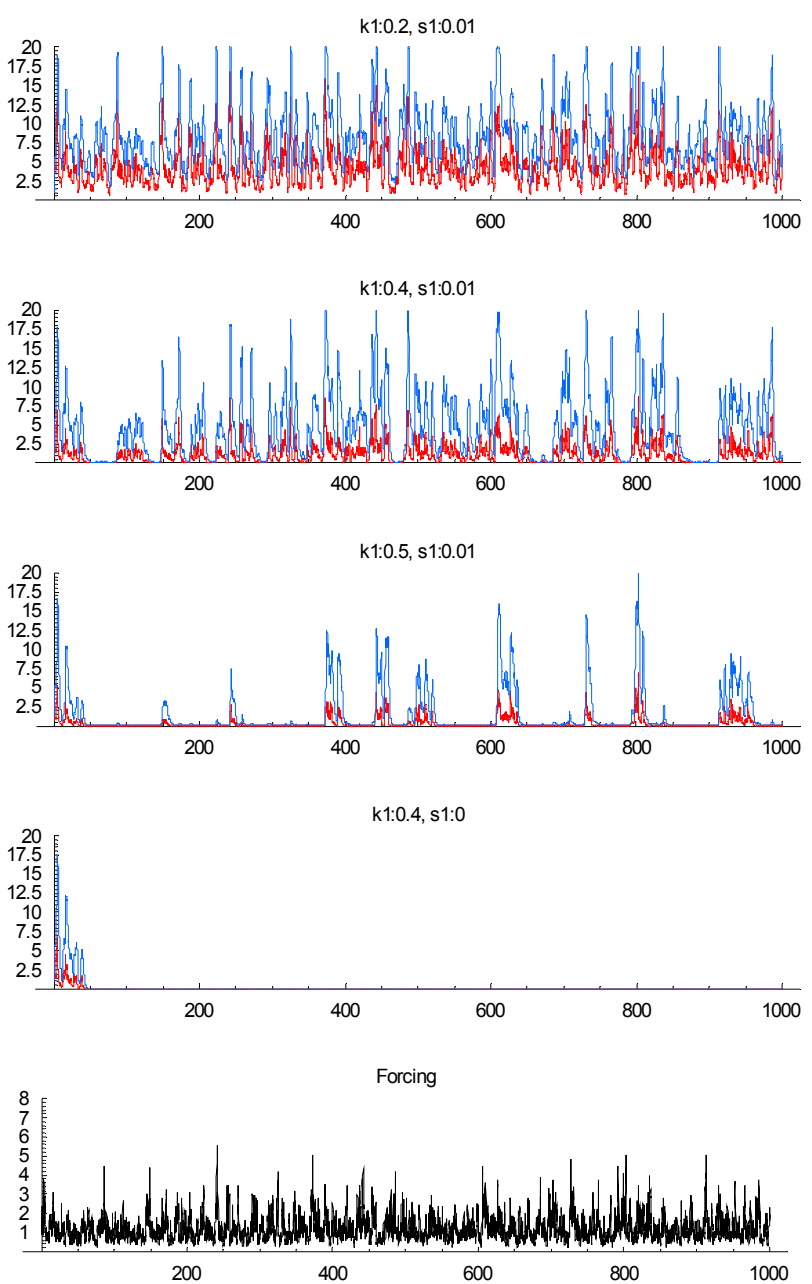

Fig. 11. Time series of $x_{1}(t)$ (red) and $x_{2}(t)$ (blue) for the two-equation model of plant $\left(x_{1}\right)$ and soil $\left(x_{2}\right)$ carbon dynamics, Eqs. (27) and (28), with parameters $q_{1}=1, q_{2}=1, k_{2}=0.1$, and $\left(k_{1}, s_{1}\right)=(0.2,0.01),(0.4,0.01),(0.5,0.01)$, and $(0.4,0)$ (top to second bottom panels). Parameters for the top panel correspond to the reference case. The bottom panel shows the forcing term $F(t)$, from Eq. (31) with $F_{0}=1, \sigma_{m}=0.5, T_{m}=1$.

system flips randomly between these two states, fluctuating around one of these points and then the other. The flips are triggered by the interaction between the forcing $F(t)$, the state $\left(x_{1}(t), x_{2}(t)\right)$ and the basin of attraction for each equilibrium point. If the system is in the active state (fluctuating near point C) and a "drought" occurs, represented by a period when $F(t)$ is anomalously low, then the system can flip into the dormant state and fluctuate around point A. Conversely, a period of anomalously high $F(t)$ can flip the system from point A to point $C$. It is not visually apparent what aspects of $F(t)$ cause the flip. This aspect of the model behaviour is reminiscent of the blooming of desert ecosystems in response to rain, interspersed with long periods of dormancy.

The third kind of behaviour is illustrated by the fourth 
panel in Fig. 11. In this case $s_{1}=0$ and $k_{1}=0.4$ (with other parameters at reference-case values), so the fourth panel is the same as the second except for the change of $s_{1}$ from 0.01 to 0 . The effect of this change is that equilibrium point $\mathrm{A}$ is now at the origin, so the first flip of the system from point $\mathrm{C}$ to point A leads to "extinction". Recovery from point A is impossible under any forcing with $s_{1}=0$.

The random, noise-driven flips between locally stable states evident in Fig. 11 are not the same as dynamical deterministic chaos, for which a paradigm is the 3-dimensional Lorenz system (Drazin, 1992; Glendinning, 1994). Deterministic chaos is exhibited by nonlinear deterministic systems with solutions which are aperiodic, bounded and sensitively dependent on initial conditions, meaning that nearby trajectories separate rapidly in time (Glendinning, 1994, p291). These properties are inherent in the system equations themselves, rather than being imposed by external random forcing or noise. There is an ongoing debate about whether external noise can induce chaos in ecological systems with otherwise stable equilibrium points. Dennis et al. (2003) argued that this is not possible, while Ellner and Turchin (2005) argued that the boundary between deterministic and noise-induced chaos is more subtle, exhibiting regions of "noisy stability", "noisy chaos", "quasi-chaos" and "noise-domination" depending on the noise level and the dominant Lyapunov exponent (the real part of the fastestgrowing eigenvalue). The debate appears to depend on the precise definition of "chaos". It is certainly important to distinguish between endogenous, deterministic chaos as in the Lorenz system and noise-induced chaos as in Fig. 11, because noise-induced chaos disappears as the noise level goes to zero whereas deterministic chaos does not.

\section{Summary and conclusions}

This paper has analysed simple models for "productionutilisation" systems, reduced to two state variables $\left(x_{1}(t), x_{2}(t)\right)$ for producers and utilisers, respectively. Two modes have been distinguished: in "harvester" systems, resource utilisation involves active seeking on the part of the utilisers (as in prey-predator systems, for example), while in "processor" systems, utilisers act as processors which passively receive material from the production part of the system. The formal expression of this distinction is that the utilisation flux $\left(g_{2}\right)$ depends directly on the utiliser component $x_{2}$ in harvester systems, for example as $g_{2}=p_{2} x_{2} x_{1}$, whereas $g_{2}$ is not dependent on $x_{2}$ in processor systems.

An idealised model of biosphere-human interactions, consisting of two coupled equations for the time evolutions of biomass $b(t)$ and human population $h(t)$, provides an example of a harvester system. This model has been analysed in two forms, a basic form in which production is constant and harvest is simply proportional to $b h$, and an extended form in which the production and harvest fluxes $\left(g_{1}, g_{2}\right)$ are both limited by biospheric resources $(b)$ at low $b$ and saturate at high $b$. The properties of these two variants of the model are somewhat different, but the following aspects are common to both: the model produces a "biosphere-only" equilibrium which is stable in the absence of humans, and a "coexistence" equilibrium to which the system is attracted whenever the initial human population is greater than zero. Trajectories in the $b h$ plane tend to the coexistence equilibrium point from any initial state with $h>0$, either without or with oscillatory behaviour manifested as decaying spiral orbits. The properties of the coexistence equilibrium can be quantified in terms of a "resource condition index" $(W)$, the ratio of the biomasses at the coexistence and biosphere-only equilibria. However, there are also some significant differences between the basic and extended forms of the model: four important ones are (1) an additional equilibrium point at the origin in the extended model; (2) different responses to declining resource condition $(W)$, the extended model being more realistic; (3) a greater tendency to strong oscillatory behaviour in the extended model than in the basic model; and (4) the possibility in the extended model that the coexistence equilibrium is unstable, leading to limit cycles at low $W$ with strong resource limitation.

An idealised model of plant and soil carbon dynamics is used as an example of a processor system. The model formulation includes a production term with a resource-limitation dependence on producer (plant carbon, $x_{1}$ ) level and a symbiotic dependence on utiliser (soil carbon $x_{2}$ ) level, together with a small constant production term $\left(s_{1}\right)$ which is independent of both $x_{1}$ and $x_{2}$. The model has three equilibrium points: a stable "active-biosphere" equilibrium, a stable "dormant-biosphere" equilibrium, and an unstable equilibrium point between them. The dormant-biosphere equilibrium is biophysically realisable only in a subset of parameter space. If the production term $s_{1}$ is zero, then the stable, dormant-biosphere equilibrium (if parameter values allow it to exist) is at the origin and corresponds to an extinction point for the system. All stable equilibria for this plant-soil carbon model are nodes, that is, they have negative, real eigenvalues so that trajectories approach them without oscillatory behaviour.

The plant-soil carbon model has been used to study the effect of random forcing of production (for example by weather and climate fluctuations). With parameter choices that allow the existence of both the active-biosphere and dormant-biosphere equilibria, the model can flip between them under the influence of random forcing, producing a bimodal behaviour in which the model fluctuates alternately around these two very different equilibrium states. It is important to distinguish this kind of externally-driven transition between states from Lorenzian chaos (Glendinning, 1994). In the Lorenzian system, random flips between states (represented by the two lobes of the Lorenz attractor) are endogenous properties of the system. In the present example, flips between states occur as the system crosses a threshold under 
the influence of external forcing, although the interactions between state, trajectory and forcing make it hard to form a simple rule for when the flip will occur.

Finally, we have highlighted a basic difference between processor and harvester forms of two-component producerutiliser systems as introduced at the start of this paper: harvester systems may exhibit oscillatory behaviour whereas processor systems do not.

\section{Appendix A}

\section{Stability properties of the basic biosphere-human model}

The basic biosphere-human model, Eqs. (11) and (12), has two equilibrium points (A and B) given by Eq. (14). From Eqs. (9) and (10), the stability properties of an equilibrium point $\left(b^{Q}, h^{Q}\right)$ are characterised by the determinant and trace of the Jacobian $\mathbf{J}$, evaluated at that point. For this model, the Jacobian is

$\mathbf{J}=\left(\begin{array}{cc}-k-h & -c b \\ r c h & r(c b-m)\end{array}\right)$

In terms of the dimensionless groups $U$ and $V$, the determinant and trace of $\mathbf{J}$ at the two equilibrium points are:

$$
\begin{array}{ll}
\text { A: } \operatorname{Det} \mathbf{J}=\frac{k^{2} V(U-1)}{U}, & \operatorname{Tr} \mathbf{J}=k\left(\frac{V(1-U)}{U}-1\right) \\
\text { B: } \operatorname{Det} \mathbf{J}=\frac{k^{2} V(1-U)}{U}, & \operatorname{Tr} \mathbf{J}=-\frac{k}{U}
\end{array}
$$

Hence, for all biophysically admissible parameter choices $(0 \leq U \leq 1$ and $0 \leq V)$, Det $\mathbf{J}<0$ at point $\mathrm{A}$ and Det $\mathbf{J}>0$, $\operatorname{Tr} \mathbf{J}<0$ at point B. Evaluating stability with Eq. (9), point $\mathrm{A}$ is a saddle point and point $\mathrm{B}$ is stable.

The two eigenvalues at each equilibrium point are

$$
\begin{aligned}
& \text { A: } \lambda_{1}=-k, \quad \lambda_{2}=k V\left(U^{-1}-1\right) \\
& \text { B: } \lambda_{1,2}=\frac{k}{2 U}(1 \pm \sqrt{1-4 U V(1-U)})
\end{aligned}
$$

The eigenvalues at point $\mathrm{A}$ are both real and of opposite sign. Inspection of Eqs. (11) and (12) (or evaluation of the eigenvectors) shows that the stable axis of this saddle point is oriented along the axis $h=0$, so that point $\mathrm{A}$ is stable if $h=0$ and unstable otherwise. The eigenvalues at point B both have negative real parts, consistent with stability. Point B is a stable focus (spiral trajectories) when $V>(4 U(1-U))^{-1}$, and a stable node otherwise.

\section{Appendix B}

\section{Stability properties of the dimensionless extended biosphere-human model}

The dimensionless extended biosphere-human model, Eqs. (20) and (21), has three equilibrium points (Z, A, B) given by Eq. (22). In terms of the resource condition index $W$ defined by Eq. (23), the Jacobian of the model is:

$\mathbf{J}=\left(\begin{array}{cc}\frac{a_{1}\left(1+a_{1}\right)}{\left(x_{1}+a_{1}\right)^{2}}-1-\frac{V\left(1+a_{2} W\right) x_{2}}{W\left(1+a_{2} x_{1}\right)^{2}} & -\frac{V\left(1+a_{2} W\right) x_{1}}{W\left(1+a_{2} x_{1}\right)} \\ \frac{V\left(1+a_{2} W\right) x_{2}}{W\left(1+a_{2} x_{1}\right)^{2}} & -\frac{V\left(x_{1}-W\right)}{W\left(1+a_{2} x_{1}\right)}\end{array}\right)$

The determinant and trace of $\mathbf{J}$ at each equilibrium point are:

Z: $\quad$ Det $\mathbf{J}=-\frac{V}{a_{1}}, \quad \operatorname{Tr} \mathbf{J}=\frac{1}{a_{1}}-V$

A: Det $\mathbf{J}=\frac{V(W-1)}{W\left(1+a_{1}\right)\left(1+a_{2}\right)}$,

$\operatorname{Tr} \mathbf{J}=-\frac{V(W-1)\left(1+a_{1}\right)+W\left(1+a_{2}\right)}{W\left(1+a_{1}\right)\left(1+a_{2}\right)}$

B: Det $\mathbf{J}=\frac{V(1-W)}{\left(W+a_{1}\right)\left(1+a_{2} W\right)}$,

$$
\operatorname{Tr} \mathbf{J}=-\frac{W\left(1+a_{1}-a_{1} a_{2}+2 a_{1} a_{2} W+a_{2} W^{2}\right)}{\left(W+a_{1}\right)^{2}\left(1+a_{2} W\right)}
$$

Using Eqs. (9) and (10) and the existence conditions $0 \leq W \leq 1,0 \leq V, 0 \leq a_{1}$ and $0 \leq a_{2}$ for biophysically admissible parameters, the following stability properties are obtained for the three equilibrium points. At point $\mathbf{Z}$ (the origin), Det $\mathbf{J}$ is always negative and $\operatorname{Tr} \mathbf{J}$ is of either sign. Therefore, point $\mathbf{Z}$ is a saddle point. Inspection of Eqs. (20) and (21) (or evaluation of the eigenvectors) shows that point $\mathrm{Z}$ is unstable with respect to an infinitesimal variation in $x_{1}$ and stable with respect to a variation in $x_{2}$, so the stable axis of the saddle point at the origin is oriented along the $x_{2}$ axis. At point $\mathrm{A}$ (the biosphere-only equilibrium), Det $\mathbf{J}$ is always negative and $\operatorname{Tr} \mathbf{J}$ is always negative. Hence this point is a saddle point. Its stable axis is oriented along the $x_{1}$ (biomass) axis, as in the basic model. At point B (the coexistence equilibrium), Det $\mathbf{J}$ is always positive and $\operatorname{Tr} \mathbf{J}$ is of either sign. Hence this point is either stable (if ( $\operatorname{Tr} \mathbf{J})<0$, evaluated at point $\mathrm{B}$ ) or unstable (if $(\operatorname{Tr} \mathbf{J})>0$ ). This leads to the criteria given in Eqs. (25) and (26).

Acknowledgements. Discussions with N. J. Grigg, C. M. Trudinger, J. G. Canadell, B. H. Walker, D. J. Barrett and J. J. Finnigan have been important in forming the ideas presented here. I am grateful to the organisers of the Oliphant Conference on Thresholds and Pattern Dynamics for the opportunity to participate, and also for support from the CSIRO Complex System Science Initiative.

Edited by: C. Hinz

\section{References}

Bolker, B. M., Pacala, S. W., and Parton, W. J.: Linear analysis of soil decomposition: Insights from the century model, Ecol. Appl., 8, 425-439, 1998.

Boyden, S.: The Biology of Civilisation, p. 189, University of New South Wales Press Ltd., Sydney, 2004.

Bridgman, P. W.: Dimensional Analysis, p. 113, Yale University Press, New Haven, CT, 1931. 
Casti, J. L.: Five Golden Rules, p. 235, John Wiley and Sons, Inc., New York, 1996.

Casti, J. L.: Five More Golden Rules, p. 267, John Wiley and Sons, Inc., New York, 2000.

Dennis, B., Desharnais, R. A., Cushing, J. M., Henson, S. M., and Costantino, R. F.: Can noise induce chaos?, Oikos, 102, 329339, 2003.

Diamond, J.: The Rise and Fall of the Third Chimpanzee, p. 360, Vintage, London, 1991.

Diamond, J.: Guns, Germs and Steel, p. 480, Vintage, London, 1997.

Diamond, J.: Collapse, p. 575, Allen Lane, Penguin Group, New York, 2005.

Drazin, P. G.: Nonlinear Systems, p. 317, Cambridge University Press, Cambridge, 1992.

Ellner, S. P. and P. Turchin: When can noise induce chaos and why does it matter: a critique, Oikos, 111, 620-631, 2005.

Flannery, T. F.: The Future Eaters, p. 423, Reed Books, Melbourne, 1994.

Fletcher, C. S., Miller, C., and Hilbert, D. W.: Operationalizing resilience in Australian and New Zealand agroecosystems. 2006. Pocklington, York, UK, International Society for the Systems Sciences. Proceedings of the 50th Annual Meeting of the ISSS, Sonoma State University, Rohnert Park, California, USA.

Glendinning, P.: Stability, Instability and Chaos: an Introduction to the Theory of Nonlinear Differential Equations, pp. 1-388, Cambridge University Press, Cambridge, 1994.
Gurney, W. S. C. and Nisbet, R. M.: Ecological Dynamics, p. 335, Oxford University Press, Oxford, 1998.

Huntley, H. E.: Dimensional Analysis, p. 158, Dover Publications, New York, 1967.

Kot, M.: Elements of Mathematical Ecology, pp. 1-453, Cambridge University Press, Cambridge, 2001.

Lotka, A. J.: Undamped oscillations derived from the law of mass action, J. Am. Chem. Soc., 42, 1595-1599, 1920.

Raupach, M. R., Barrett, D. J., Briggs, P. R., and Kirby, J. M.: Simplicity, complexity and scale in terrestrial biosphere modelling, in: Predictions in Ungauged Basins: International Perspectives on the State-of-the-Art and Pathways Forward (IAHS Publication No. 301), edited by: Franks, S. W., Sivapalan, M., Takeuchi, K., and Tachikawa, Y., 239-274, IAHS Press, Wallingford, 2005.

Trudinger, C. M., Raupach, M. R., Rayner, P. J., Kattge, J., Liu, Q., Pak, B. C., Reichstein, M., Renzullo, L., Richardson, A. E., Roxburgh, S. H., Styles, J. M., Wang, Y. P., Briggs, P. R., Barrett, D. J., and Nikolova, S.: The OptIC project: an intercomparison of optimisation techniques for parameter estimation in terrestrial biogeochemical models, JGR Biogeosci., in press, 2007.

van Kampen, N. G.: Stochastic Processes in Physics and Chemistry, p. 419, North-Holland, Amsterdam, 1981.

Volterra, V.: Variazioni e fluttuazioni del numero d'individui in specie animali conviventi, Mem. Accad. Naz. Lincei, 2, 31-113, 1926.

Wirtz, K. W. and Lemmen, C.: A global dynamic model for the neolithic transition, Clim. Change, 59, 333-367, 2003. 